\title{
ENJEUX SOCIO-CULTURELS DE LA RÉGULATION MARCHANDE DES MÉDIAS AUDIOVISUELS
}

\author{
Emmanuel Belin ${ }^{1}$
}

L'évidence avérée d'une inefficience des systèmes économiques basés sur la gestion politique de la rareté doit sans doute se comprendre moins comme une leçon de l'histoire que comme un événement ramassant autour de quelques images frappantes les schémas de légitimité implicites qui régissaient déjà bien avant la chute du mur les choix politiques dans les démocraties occidentales. La construction européenne, presque réduite à celle d'un marché unique, est un indicateur de cette référence de fait à l'idéologie néolibérale. Ce qui caractérise ce processus, ce n'est sans doute pas tant la conviction d'une efficacité supérieure de la régulation des activités par le marché que celle d'une antériorité de ce type d'équilibrage sur les autres formes d'intervention. Dès lors qu'existent une rareté et une utilité, et quelle que soit la nature de celles-ci, l'échange marchand est considéré comme l'arrangement permettant le plus sûrement d'atteindre des équilibres initiaux, le cas échéant compensables par d'autres logiques portant sur les externalités des solutions ainsi réalisées. Sous-jacent à cette manière de procéder apparaît le postulat

1 Aspirant au Fonds National de la Recherche Scientifique (FNRS).

Recherches en communication, $\mathrm{n}^{\circ} 4,(1995)$. 
inquestionné de la pertinence d'une focalisation sur tous les aspects qui, parce que mesurables en termes monétaires, forment la dimension proprement économique des phénomènes régulés, comme si celle-ci, en toute circonstance, devait être considérée comme le cœur, l'essentiel du problème -l'externalité économique devenant synonyme de secondarité ${ }^{1}$. Cette définition de la réalité, ancrée elle-même dans l'histoire philosophique de la modernité, conduit à admettre une comparabilité illimitée de tous les types d'utilité, via le médium monétaire, et donc la possibilité de les traiter tous de la même manière.

En matière de services publics, la politique menée par les pouvoirs nationaux ${ }^{2}$, mais surtout par la Commission européenne, semble avoir longtemps été uniquement orientée dans ce sens. Pour P. Bauby et J.-C. Boual, "la Commission manifeste la volonté de considérer les services rendus par les services publics comme des marchandises identiques aux autres, sans tenir compte de leurs spécificités, de l'histoire, des conditions sociales, ni des besoins sociaux auxquels répondent les services publics"3. Dans le cas précis de l'audiovisuel, il apparaît que l'exclusivité du discours néo-libéral a été sérieusement défiée à partir de 1989. Cependant, comme le montre A. Lange ${ }^{4}$, le passage du livre vert "Télévision sans frontières" de 1984, qui considère les activités de diffusion comme de simples services, à la directive de 1989, où est admis le caractère spécifique des biens

1 Cette problématique constitue le noyau de la socio-économie qui, partant de la notion d'encastrement de Polanyi et de Granoveter, débouche sur une reconsidération des paradigmes économiques classiques, vus comme transposition d'une idéologie posant la rareté matérielle comme fait originel. Ce courant indique que la place des "externalités" est variable historiquement et synchroniquement, la dimension économique, dans certaines circonstances, étant à considérer comme accessoire, secondaire par rapport aux autres aspects de la réalité régulée. Cf. A. CAILlÉ, "Faut-il créer une nouvelle discipline dans les sciences sociales, et laquelle?", Revue du MAUSS, $\mathrm{n}^{\circ} 15-16$, an. inc.***, p. 11-42.

2 Yves Achille souligne bien la responsabilité des États de différents pays d'Europe, non seulement dans la déréglementation du marché, mais aussi et surtout dans la manière dont ce processus s'est déroulé. Si des politiques plus intelligentes avaient été mises en place, des équilibres entre chaînes privées et publiques auraient pu être trouvés. (Y. ACHILLE et J. IBANEZ BUENo, Les télévisions publiques en quête d'avenir, Grenoble, Presses Universitaires de Grenoble, 1984, en particulier p. 63 à 94).

3 P. Bauby et J.-Cl. Boual, "Quels services publics demain en France, en Europe", in P. BAUBY et J.-Cl. BouAL (eds.), Les services publics au défi de l'Europe, Paris, Les Éditions ouvrières, 1993, p. 11-47.

4 A. LANGE, “"Descartes, c'est la Hollande». La Communauté Européenne : culture et audiovisuel", Quaderni, n¹9, 1993, p. 83-116. 
culturels, concerne surtout le plan des principes, et s'avère sujet à controverses pour ce qui est de son application. Enfin, le traité de Maastricht de 1992 reconnaît une compétence communautaire spécifique en matière culturelle - même si, à de nombreux égards, les modalités de cette compétence sont critiquables en tant qu'axées sur une conception trop substantialiste et républicaine de l'identité européenne ${ }^{1}$. Force est pourtant de constater que de facto, l'introduction de la logique marchande dans la sphère médiatique, dès lors que celleci s'avère profitable, est une tendance historique constante de la seconde moitié du siècle ${ }^{2}$. Au-delà des déclarations de principe, souvent intuitives, il importe de s'interroger sur la portée réelle de cette absorption par l'économique. C'est de cette interrogation que nous partirons. Il importe à la fois d'ancrer une évaluation de la régulation dans une étude des effets et d'orienter cette réflexion analytique par une critériologie fondée dans cette réflexion normative sur les modes de régulation.

\section{Spécificité des produits audiovisuels par rapport au marché}

Trois caractéristiques des produits audiovisuels justifient qu'on interroge l'adéquation de leur régulation marchande : leur nature physique, leur spécificité économique et leur statut socio-politique. La première particularité tient à la nature immatérielle du bien échangé. Certes, celui-ci se réalise toujours grâce à des supports matériels, mais ces appareils interviennent pour une faible part dans la détermination de la valeur d'usage du produit télévisuel, l'utilité résidant plutôt dans le potentiel d'orientation subjective contenu dans les informations diffusées. Comme le montre $\mathrm{A}$. Mayère, cet aspect ne suffit pas à arracher le bien informationnel à la sphère de la marchandise, à condition toutefois de penser à nouveaux frais cette notion abusivement réduite aux biens matériels :

1 Ce débat est abordé par J.-M. FERry, "Pertinence du post-national", Esprit, $\mathrm{n}^{\circ} 176$, 1991, p. 80-93.

2 E. WITTE, De breedte van het scherm. Pleidooi voor de openbare televisie, Antwerpen, Icarus, 1994, p. 12 et svtes. Certes, la dimension marchande est présente dans la diffusion des biens culturels depuis bien avant. Cependant, l'irruption de cette dimension comme mode légitime d'appréhension, y compris au niveau de la production, est une tendance propre à notre siècle. 
ceci amène à reconsidérer la valeur d'usage non plus du point de vue du produit, comme qualité de ses propriétés intrinsèques, mais du point de vue de son utilisateur, dans l'utilisation qu'il fait de ce produit, c'est-à-dire dans son mode d'activation des propriétés du produit en fonction de ses finalités et contraintes, selon une temporalité et dans un espace fonction de ses besoins ${ }^{1}$.

Quoique se rapportant à un contexte différent -celui de l'information comme bien de production et non comme bien de consommation-, un tel élargissement met en relief trois apprêts du bien culturel nécessaires pour lui permettre d'accéder à cette catégorie. Le premier concerne la création de la rareté : la nature immatérielle du transfert de propriété (diffusion) et la non exclusivité de l'appropriation ${ }^{2}$ des biens publics que sont les émissions télévisées impliquent qu'une fois le programme produit, sa rareté soit artificiellement construite par un dispositif technique de codage. Le second est la spécification du bien, sa disponibilité au moment du contrat de transfert : il s'agit en effet que l'utilisateur puisse disposer de l'information indispensable à l'évaluation anticipative de l'utilité du bien qu'il convoite. Cette transparence est relativement peu problématique pour des produits matériels simples, mais s'abstrait lorsqu'il s'agit de biens dont la mise à disposition ne se réalise pas instantanément. Cela permet de comprendre une tendance récente à la multiplication des chaînes thématiques commerciales, tant sur le réseau câblé que via satellites, qui permettent une visibilisation accrue de la spécificité programmatique de chaque canal $^{3}$. La troisième exigence enfin tient à

1 A. MAYÈRE, Pour une économie de l'information, Paris, C.N.R.S., 1990, p. 132.

2 Même si cette non-exclusivité est seulement partielle, puisque la réception par autrui d'une information n'est pas sans effet sur l'utilité qu'a pour moi cette information. Cette nuance, capitale lorsqu'il s'agit d'information intervenant dans le processus de production, est moins pertinente quand on s'intéresse à la production audiovisuelle à des fins de consommation. Dans ce cas précis, il semble même que le fait que l'information ou le produit de divertissement soit diffusé, accessible à d'autres récepteurs, soit un facteur d'augmentation de l'utilité de ce bien, soit qu'il renforce le sentiment d'une participation à la vie collective comme le montre Daniel Dayan au sujet de la télévision interruptive (D. DAYAN et E. KATZ, Media events; the live broadcasting of history, Cambridge, Harvard University Press, 1994), ou encore qu'il permette une participation effective aux conversations de la vie sociale (p. ex. W. A. GAMSON, Talking Politics, Cambridge, Cambridge University Press, 1992 ou I. HAGEN, "Expectations and consumption patterns in TV news viewing", Media, Culture and Society, vol. 16, 1994, p. 415428).

3 D. Wolton, Éloge du grand public, Paris, Flammarion, 1990. 
l'impossibilité, vu cette fluence du produit, d'une appropriation totale, exempte de toute intervention du producteur dans la consommation ou la réalisation de l'utilité : cette persistance inévitable de la relation limite en effet considérablement la liberté d'utilisation intrinsèquement liée à la notion de propriété. Deux procédés permettent de limiter cette dépendance : la rediffusion périodique des programmations sur des tranches horaires décalées d'une part ${ }^{1}$, et le multiplexing d'autre part, qui permet grâce à l'amélioration considérable des performances du réseau câblé une interactivité accrue, brouillant la frontière entre médias de diffusion et de transmission.

La complexité de cet appareillage, liée aux caractéristiques intrinsèques du produit télévisuel, débouche sur une triple particularité des marchés qui s'y rapportent. La première découle directement du caractère impersonnalisable des produits de diffusion : il en résulte en effet que les coûts de production sont quasiment indépendants de la quantité offerte, mesurée par le nombre de récepteurs ${ }^{2}$. D'où une profitabilité extrême dès qu'un large public est atteint, ce qui fait du secteur un lieu particulièrement attrayant pour les investisseurs, et dès lors un enjeu économique de première importance. Cette importance d'une maximisation de l'offre conduit à des conséquences inattendues eu égard à la seconde particularité d'une part substantielle des activités économiques liées à la diffusion : l'inscription des offreurs dans un marché à produit dual. Les dispositifs techniques de marchandisation des produits diffusés ne sont en effet apparus que très tardivement dans le développement du secteur audiovisuel. Ce décalage explique que l'extension se soit opérée au travers d'autres logiques, et notamment dans le cas de la distribution commerciale, celle du financement par la publicité, introduisant le diffuseur dans une relation triadique entre lui, l'annonceur et son public.

Le premier marché auquel les médias participent est celui du bien, le produit médiatique (...). Le second marché auquel participent beaucoup de médias est le marché de la publicité. Bien que certains observateurs peuvent conclure informellement que les médias vendent de l'espace ou du temps à l'ache-

1 B. Miège, P. Pajon et J.-M. SAlaun, L'industrialisation de l'audiovisuel. Des programmes pour les nouveaux médias, Paris, Aubier, 1986.

2 R. G. PICARD, Media economics. Concepts and issues, Newbury Park-London-New Delhi, Sage, 1989, p. 66. 
teur de publicité, une explication plus précise et descriptive est que les médias vendent l'accès au public à des annonceurs ${ }^{1}$.

Cette articulation introduisant un lien direct entre le public atteint et les revenus financiers conduit logiquement à une réduction extrême du prix à payer pour le spectateur, tendant le plus souvent vers la gratuité complète. L'antécédence historique de ce dispositif institutionnel sur l'appareillage technique d'autonomisation du produit télévisuel est un fait capital dans la compréhension de leur logique de fonctionnement. Elle a en effet structuré en profondeur le rapport aux chaînes commerciales, assurant la création d'une culture médiatique dont la possibilité matérielle de la télévision payante ou du pay-perview n'élude que très partiellement l'impact. La troisième caractéristique, enfin, découlant des précédentes est la propension du secteur à l'intégration, tant horizontale que verticale. La production et l'échange de programmes est un moyen de financement alternatif au paiement par le téléspectateur, y compris dans les services publics ${ }^{2}$ outre qu'il permet de garantir de manière précise l'attractivité des programmes pour les annonceurs soucieux de maîtriser l'environnement de leurs messages promotionnels. La complexité technique découlant de l'inadaptation matérielle du produit à la logique de la marchandise a, parallèlement, pour résultat la connotation extrêmement capitalistique du secteur et favorise l'émergence de consortiums. Enfin, la dépendance des producteurs et des diffuseurs à l'égard d'infrastructures extrêmement onéreuses -qu'il s'agisse de satellites de télécommunication ou de réseaux câblés- conduit immanquablement à des tentatives d'intégration verticale pouvant déboucher sur des situations oligopolistiques ou monopolistiques dès lors que l'accès au marché est conditionné matériellement par l'affiliation à une de ces structures de médiation ${ }^{3}$.

Le troisième aspect diacritique du marché des médias concerne le statut socio-politique des biens concernés. Selon la théorie libérale, la régulation marchande aboutit à une convergence entre valeurs

1 Ibidem, p. 17-18 (traduit par nous).

2 J. G. BLumLer, "Meshing Money with Mission: Purity versus Pragmatism in Public Broadcasting", European Journal of Communication, vol. 8, 1993, 403-424, p. 414 et sv.

3 Rappelons que la situation décrite concerne seulement la logique du marché à l'état pur. En réalité, dans la plupart des pays européens, ces infrastructures sont encore propriété publique -même si les tendances européennes remettent en question cette organisation. 
d'usage et d'échange en même temps qu'elle mène à une amélioration de la qualité des produits. Or, dès qu'il s'agit de biens culturels, la question de l'évaluation de la qualité devient un problème philosophique et esthétique complexe ${ }^{1}$, consacrant un caractère relatif ${ }^{2}$ du jugement à cet égard. Dans le débat sur l'impact socio-politique d'une marchandisation des médias, cette qualité peut donner lieu à deux interprétations: intrinsèque (textuelle) et extrinsèque (contextuelle et intertextuelle). Ces deux catégories sont relatives au potentiel d'autonomie socio-politique véhiculé par le flux médiatique dans son ensemble. Ce potentiel sera dit intrinsèque lorsqu'il concernera les produits pris comme tout, et extrinsèque lorsqu'il résultera de la mise en relation des produits les uns avec les autres par une activité explicite du récepteur. Outre ces deux domaines relatifs aux effets directement socio-politiques, nous tiendrons compte de l'impact indirect en termes d'épanouissement individuel. Pour l'instant, contentons-nous d'observer que, dans une logique libérale pure, tous ces effets sont à comprendre comme des externalités et se résorbent dans l'expression quantitative de la demande ${ }^{3}$. Or, la plupart des commentateurs semblent considérer que cette résorption est porteuse d'effets néfastes sur le plan socio-politique ${ }^{4}$. Pour Boris Libois, la philosophie politique a produit divers types d'argumentation pour justifier la spécificité de la sphère médiatique, et donc la nécessité d'adopter pour la réguler des formes appropriées :

"Pour les penseurs libertariens et libéraux", écrit-il, "les médias sont la concrétisation logique des droits naturels de l'individu que sont la liberté d'expression et la liberté de la presse. A l'inverse, pour les théoriciens de la «responsabilité sociale de la presse» et du «droit à l'information», les médias remplissent une fonction publique et sont des institutions

1 Cf. K. Chr. SchrøDER, "Qualité culturelle : la poursuite d'un fantôme ?", Hermès, $\mathrm{n}^{\circ} 11-12,1992$, p. $95-110$.

2 Et non forcément relativiste, qui n'est qu'une des positions possibles dans le débat. Dire que la qualité est relative signifie ici qu'elle ne se mesure qu'en fonction d'un contexte normatif et pragmatique donné, et non par un critère univoque et valable en toute circonstance.

3 Cf. B. Perret et G. Roustang, L'économie contre la société. Affronter la crise de l'intégration sociale et culturelle, Paris, Éd. du Seuil, 1993, p. 168.

4 B. Libols, "Redéfinir l'audiovisuel de service public", Actes des Assises de l'Audiovisuel de service public, Bruxelles, Diffusion Alternative, 1993. 
sociales essentielles au bon fonctionnement de la démocratie"l.

Cependant, ces conceptions normatives n'ont jamais été élaborées sur base d'une étude empirique approfondie de la situation concrète où se négocient entre un spectateur et un texte les significations pragmatiques d'un produit culturel -domaine laissé généralement à l'approche sémiologique.

\section{La nécessité d'une évaluation réflexive des critères d'analyse empirique}

Le bouclage logique entre discours analytique et normatif dont il était question plus haut se traduit, lorsqu'il s'agit de réfléchir à l'impact socio-politique réel des médias, par la nécessité de définir à partir d'une conception normative de l'espace public le cadre théorique qui nous guidera dans la définition des voies pertinentes d'investigation. Ce scrupule est rarement de mise chez les chercheurs de sociologie de la communication. Le plus souvent, les grilles de lecture qu'ils appliquent à la réalité s'ancrent en conséquence dans un fonds inquestionné d'évidences normatives; or, les sociologues sont également des intellectuels, et à ce titre porteurs de logiques normatives très spécifiques, surtout précisément dans le champ de la culture et de sa diffusion. Ces normes, valeurs et représentations propres, si elles ne font pas l'objet d'une élucidation réflexive, tendent à apparaître comme une segmentation adéquate et universelle du réel social, alors qu'elles n'en sont qu'une version particulariste. In fine, cette carence peut conduire à l'occultation pure et simple de formes nouvelles, donc à un parti pris conservateur ${ }^{2}$. Concrètement, deux conceptions normatives nous semblent devoir être explicitées impérativement : la définition normative de la liberté et celle de la publicité. Le premier débat est classique en philosophie politique : il voit s'opposer les partisans d'une définition de la liberté liée à la propriété privée à ceux qui en prônent une conception enracinée dans la liberté

1 B. LiBors, Éthique de l'information. Essai de déontologie journalistique, Bruxelles, Éditions de l'Université Libre de Bruxelles, 1994, p. 24.

2 A cet égard, il n'est sans doute pas exagéré d'affirmer que la majorité des sociologues raisonnent encore dans un paradigme critique tel que celui développé par l'École de Francfort, notamment par Adorno et Horkheimer. 
d'expression. Il se relie à la problématique des effets des médias par l'intermédiaire de la question de l'accès aux médias, qui elle-même renvoie à celles du pluralisme et du droit à l'information : le problème est dans ce cas de définir clairement ce qu'on entend par "pluralisme", dans l'optique mentionnée d'une théorie des effets extrinsèques. Le second enjeu philosophique sous-jacent concerne la dichotomie classiquement admise entre le public et le privé ; l'hypothèse que nous soutiendrons ici est que le concept normatif de publicité auquel se réfèrent les sociologues a été produit dans un contexte historique de rapport entre l'économique et le culturel, aujourd'hui pour une large part dépassé. Ce second enjeu touche plutôt aux caractéristiques intrinsèques des flux, les évaluant sur une définition implicite de la "qualité" définie comme une articulation "correcte" des deux registres.

La plupart des travaux de sociologues traitant de la question du pluralisme se sont construits en opposition à la simplification de certains libéraux qui le considèrent comme l'expression sur le plan des contenus diffusés de la liberté d'accès aux médias, et donc comme la résultante exclusive d'une déréglementation totale du marché. Selon ce discours, en effet, le marché libre conduit par définition au forum public, l'intervention étatique étant à considérer comme une forme de censurel. Il s'est alors agi pour les sociologues de démontrer que le marketplace of ideas indispensable au fonctionnement démocratique n'est pas possible sans intervention étatique ${ }^{2}$; or, cette démarche implique une évaluation du caractère pluraliste d'une programmation, dont le critère ne fait pas forcément consensus. On

1 L'argument selon lequel l'absence d'intervention étatique découle de la liberté de la presse, elle-même à comprendre comme conséquence de la liberté d'expression, et de ce fait antérieure à tous les autres droits est contesté philosophiquement par B. Libois, pour qui cette association résulte d'un amalgame entre un droit individuel fondamental et une fonction publique. Pour lui, "seule la dimension politique de la liberté d'expression jouirait d'un statut particulier au sein des autres droits et libertés. De plus, la liberté de la presse devrait être comprise non pas en tant que droit individuel mais en tant que fonction publique, en étroite articulation avec cette reconnaissance spécifique de la liberté d'expression" (Éthique de l'information, op. cit., p. 39). Dominic Caristi ("The Concept of a Right to Access to the Media: a Workable Alternative", Suffolk University Law Review, $\mathrm{n}^{\circ} 22,1988$, p. 103-128) aboutit à un résultat quelque peu différent : constatant que le marché à lui seul conduit inévitablement à une opposition entre le droit de propriété et la liberté d'expression, il conclut au caractère indispensable d'un forum public subventionné par l'État.

2 D. CARISTI, op. cit. 
peut par exemple, sans entrer dans les détails, distinguer des approches capitalistiques (lutte contre la concentration), génériques (nécessité d'une diversification des genres télévisuels), méthodologiques (en assurant, par exemple, la multiplicité des points de vue sur chaque thème d'information ou, au niveau de la collecte des données, la multiplication des sources), institutionnel (parité au sein des conseils d'administration ou d'organes de décision) ou encore référentiel (alternance des références théoriques des analystes) ${ }^{1}$.

Au-delà de la pluralité des conceptions, deux remarques peuvent être formulées. La première concerne la logique négative qui y transparaît: en l'absence d'une définition unifiée du pluralisme, c'est implicitement à une désignation apophatique qu'il est fait référence : on stigmatise avant tout une forme de standardisation, de normalisation des produits médiatiques, on cherche à débarrasser la présentation médiatique du biais consensualiste, afin de rendre plus visible le processus de construction, de mise en scène qu'inévitablement toute médiation produit, mais également pour permettre à chaque citoyen de confectionner lui-même, par un travail réflexif, son opinion à partir des fragments et points de vue qui lui sont soumis. Il est significatif à cet égard de constater que ces théories du pluralisme ne se construisent plus en opposition à la notion de tutelle étatique, mais bien contre deux formes de "totalitarisme" privé: le monopole d'une part, et la rationalité économique d'autre part (excepté, cela va sans dire, pour le pluralisme capitalistique). Cela ne signifie pas qu'on puisse sur le plan normatif prôner un retour au monopole public, mais plutôt qu'il faut inventer des modes de régulation nouveaux. Une seconde remarque concernant les conceptions implicites du pluralisme qui guident ces recherches empiriques porte sur la déconnexion de ces catégories de toute réflexion réelle sur l'effet concret. Y transparaît la conception libérale d'un téléspectateur adulte, capable de tirer parti des vertus émancipatrices de la confrontation des points de vue, d'agencer ses soirées selon divers genres télévisuels en plein accord avec sa conception personnelle de la "vie bonne". Or, comme l'ont montré les théories des effets limités, notamment en termes de $t w o-$ step flow et de uses and gratifications, mais aussi les recherches ethnographiques ou expérimentales sur l'activité de réception, une version du pluralisme élaborée en termes de «fonction publique des

1 Ces diverses formes ont fait l'objet d'articles ou de livres dont il ne nous est pas possible, pour des raisons de place, de citer ici la référence. 
médias» ou de «droit à l'information» ne peut prendre un réel efficace qu'à condition de se détacher d'un modèle linéaire de la communication, où le message est le lieu fermé du sens transmis codé d'un émetteur à un récepteur ${ }^{\prime}$, à une sémantique pragmatique étudiant les dispositifs d'énonciation comme lieu de transaction ou de coconstruction où s'articulent un potentiel de sens et un contexte propre au récepteur. Certes sans tomber dans un travers communautarien, il importe, dès lors qu'on tente de comprendre les effets socio-politiques des médias, de prendre en compte cette insuffisance d'une centration exclusive sur l'émetteur.

Où la question libérale du "pluralisme" posait des problèmes avant tout conceptuels, celle de "qualité" implique généralement un engagement moins théorique ${ }^{2}$. Nous ne nous attacherons évidemment pas à une définition absolue de la qualité : il s'agit pour nous plus prosaïquement d'évaluer les potentialités d'autonomisation, sur base d'un concept normatif d'espace public, contenues dans la mise en forme par les médias de la réalité dont ils parlent. Peut-être parce qu'elle semble moins substantielle, moins lisible en termes de critères et de définitions positives, la notion de qualité plus que celle de pluralisme s'est élaborée en général dans une réflexion intégrant d'emblée la dimension des effets, dans un souci de prendre en compte l'inscription sociale des messages diffusés. Les théories des effets des médias sont multiples, et il n'entre pas dans notre intention d'en présenter un panorama ${ }^{3}$. En guise de clarification, nous nous contenterons d'une classification en trois catégories : les théories des effets limités (usages et gratifications, two-step flow of communication, théories de la réception), les théories des effets puissants (agendasetting et agenda-building, priming, framing, prime definition, spirale du silence) et les théories de effets profonds (approches postmodernes en termes de flux médiatiques, de définition des cadres de l'expérience physique et sociale, de visibilisation de la norme).

Les deux premiers courants de recherche empirique (effets limités et effets puissants) établissent généralement un lien entre les

1 J.-P. MEUNIER, "Nouveaux modèles de communication, nouvelles questions", Recherches Sociologiques, XXI, n³, 1990, p. 267-288.

2 Cf. K. Chr. SCHRøDER, op. cit.

${ }^{3} \mathrm{Ce}$ travail de dépouillement littéraire a déjà été réalisé par plusieurs auteurs, notamment D. MCQUAIL, Mass Communication Theory: An Introduction, 2nd edition, London, Sage, 1987; J. LAZAR, Sociologie de la communication de masse, Paris, Armand Colin, 1991. 
médias et la participation à la vie politique. Pourtant, tout en offrant des instruments solides, ils apparaissent à maints égards insuffisants pour peser l'impact réel du véritable bain médiatique dans lequel flotte l'individu contemporain sur la constitution et le fonctionnement de l'espace public. Premièrement parce que, à la mode anglo-saxonne, ils consistent le plus souvent en une élaboration patiente et méticuleuse de quelques hypothèses de base, portant sur les aspects les plus mesurables, les plus tangibles des productions médiatiques. À cet égard, on peut dire qu'ils sacrifient l'heuristique à l'instrument de mesure. Deuxièmement, ces recherches restent, en tout cas pour celles qui s'inscrivent dans le courant de l'agenda-setting, cantonnées à des définitions très restreintes de l'impact socio-politique, ici aussi d'ailleurs par souci de mesurabilité: quand ce ne sont pas simplement les résultats des scrutins électoraux qui sont utilisés comme variables dépendantes, ce sont des mesures chiffrées de concordance des agendas; notons toutefois que des recherches plus qualitatives ont été réalisées en termes de priming et de framing. Troisièmement, ces différents paradigmes s'attachent prioritairement à un seul aspect des émissions télévisées: les informations. Cette focalisation s'explique par la définition extrêmement restrictive qu'elles donnent de la participation à la vie publique. Il semble pourtant évident que des effets puissants soient à découvrir au-delà d'une telle segmentation. Quatrièmement enfin, ils se limitent presque toujours à une réflexion en termes de possibilisation de la participation politique, et non en termes d'effets réels à long terme -ou, pour le dire autrement, sur le potentiel d'autonomisation du public plutôt qu'au passage à l'acte, qui requiert des formes spécifiques d'information ${ }^{1}$. Ces quatre remarques plaident pour un élargissement de la problématique, fût-ce au prix d'un amenuisement de la base empirique, ou d'un changement de nature du rapport scientifique au réel. De manière générique, nous avons cru pouvoir rassembler ces approches dans un "paradigme des effets profonds", prenant comme variable indépendante le flux médiatique dans son ensemble, le rapport au monde que promeut sa présence et sa substance, les structures de sens sous-jacentes, et comme variable dépendante le type de rapport à la norme, au public, au collectif, à l'historicité. Les questions qui se poseraient dans ce cas

1 Cf. J. D. KeEFer, "The News Media's Failure to Facilitate Citizen Participation in the Congressional Policymaking Process", Journalism Quarterly, n²0/2, 1993, $412-424$, p. 413. 
nous paraissent renvoyer à des enjeux plus importants : quelle est la forme d'expérience des mondes objectif, intersubjectif et subjectif promue par les médias ? Quelle conception de l'histoire est véhiculée ? Quelle représentation de la norme? Quelle articulation du privé et du public ? De l'Autre et du Même ? Quelle construction identitaire : substantielle ou processuelle ? Quelles sont les formes de rationalité, de logique mises en œuvre par ces médias ? Quelle mise en forme du désir et de la satisfaction ? Ce sont là les questions réellement importantes, au-delà des effets directement cernables en termes statistiques.

Sur ces questions, les discours scientifiques laissent généralement place aux discours d'intellectuels; se côtoient des approches en termes esthétiques, phénoménologiques destinées à rendre compte de l'expérience télévisuelle en soi d'une part, et d'autre part des considérations sociologiques et philosophiques portant sur les mutations de la modernité, renvoyant le plus souvent aux premières en guise d'invocation. Quelques hypothèses ont été posées, notamment en termes de logique intrinsèque du médium, dans une perspective mcluhanienne ou en référence aux formes rhétoriques utilisées par les médias. Dans la perspective d'une réflexion normative sur la régulation des médias, la question pertinente est celle d'une hypothétique influence de la marchandisation du produit médiatique.

\section{Conséquences socio-politiques de la marchandisation}

L'analyse des médias dans le contexte d'une réflexion sur les modes de régulation impose donc un carcan très précis à la recherche : d'un côté, l'impact de la privatisation ou de l'introduction de logiques marchandes ne nous intéresse que dans la mesure où celui-ci est susceptible d'influencer les variables que nous avons estimées pertinentes eu égard à un concept normatif d'espace public; d'un autre côté, parmi toutes les dimensions possibles des relations entre l'avènement d'une "société de communication" et les "mutations de l'historicite", ne sont pertinentes à nos yeux que celles qui touchent au mode de régulation de cette sphère d'activité. Au sein de ce carcan, les logiques décelées ont une triple fonction potentielle au sein du débat public: d'abord, elles cherchent à donner une mesure du danger éventuel qu'on court en laissant au libre jeu du marché le soin de 
réguler la production télévisuelle, et donc servent d'argumentation possible pour une intervention, par exemple sous une forme procéduralisée; ensuite, elles établissent une série de repères utiles dans l'intervention, tant il est vrai que réguler un secteur n'a de sens que quand on s'en prend à ce qui dans ce secteur relève d'enjeux sociaux et politiques cruciaux -du point de vue d'une régulation procédurale, c'est la question de l'ordre du jour des instances d'intervention qui est ici concernée; enfin, les éléments rassemblés au cours de cette enquête pourront servir à dresser un argumentaire, à instruire le débat au sein des groupes de décision, à étoffer le discours des représentants du monde de la recherche en tant que participants aux discussions.

\subsection{Deux paradigmes: intentionnel et systémique}

Selon qu'on mette en avant les risques liés à l'émergence de monopoles résultant de l'intégration horizontale et verticale de l'offre télévisuelle ou qu'on insiste sur les logiques de fonctionnement ellesmêmes du marché, liées notamment à la dépendance à l'égard de la publicité, on aura tendance à lire les évolutions du produit proposé en termes volontaristes ou systémiques. Si les approches radicales en termes de "conspiration" des grands propriétaires soucieux d'endormir les masses" sont aujourd'hui en voie de disparition', la littérature scientifique recourt encore volontiers à des paradigmes indiquant la prépondérance des stratégies politiques des acteurs dans la détermination du rôle idéologique des médias. Il en est ainsi des paradigmes de la prime definition de J.S. Hall2, selon lesquels le mode de fonctionnement des médias confère à certaines sources, et particulièrement au discours gouvernemental, une prédominance de fait ancrée dans une précédence: c'est à lui que revient en effet la fonction de définir les "armes du duel", ce par rapport à quoi les points de vue alternatifs

1 James Curran, toutefois, pose la question en termes organisationnels à partir d'une étude de cas: il montre comment des pressions ont été exercées sur les journalistes par les propriétaires à l'occasion d'une controverse sur le Greater London Council ("Culturalist Perspectives of News Organizations: A Reappraisal and a Case Study", in M. FERGUSSON (ed.), op. cit., p. 114-134. Les interventions publiques de Mgr Léonard, réclamant une plus grande "docilité" des chroniqueurs religieux du groupe de presse dont il est un des dirigeants, donnent également à penser que les pressions directe sont moins anecdotiques qu'il n'y paraît.

2 Cf. J. St. HALL, "Cultural studies: two paradigms", Media, Culture \& Society, 2 (1982), p. 33-48. 
devront se construire. Sans jeter aux oubliettes l'approche volontariste, nous nous intéresserons pour l'heure surtout au second paradigme, notamment parce qu'aborder le débat en ces termes pourrait nous indiquer l'insuffisance d'une politique de lutte contre les monopoles, sans nier son éventuelle pertinence. Dans notre raisonnement, la notion d'auto-promotion jouera le rôle principal ; nous tenterons de montrer comment ce caractère auto-promotionnel du discours médiatique peut être mis en rapport avec sa marchandisation, pour ensuite étudier les implications de cette rhétorique dans le cadre d'une théorie des effets puissants (agenda setting) et d'une théorie des effets profonds (rapport au désir).

\subsection{Marchandisation et discours auto-promotionnel}

Comme l'indique F. Mariet, la chasse aux taux d'audience ne caractérise pas seulement les chaînes financées par la publicité : même les chaînes publiques, dès qu'elles dépendent pour une partie de leur financement des recettes publicitaires, doivent se définir en fonction de cette contrainte. Le GRP (Gross rating point) donne sur base d'un spot de 30 secondes le rapport entre un franc investi par l'annonceur et le nombre de téléspectateurs touchés. Il "officialise l'interaction entre l'Audimat et le financement des chaînes et surtout la consolide en termes de système de référence et de caution. On ne saurait trop insister sur le rôle capital du GRP dans le fonctionnement des chaînes. De fait, c'est lui qui règle la vie de la chaîne, et notamment la vie des programmes"l. Il est donc capital, dans un milieu concurrentiel, de parvenir à toucher le public le plus large possible afin d'attirer les fonds des annonceurs.

On peut ici noter une différence importante entre les chaînes financées par leurs auditeurs grâce à des dispositifs techniques de spécification du bien, et celles qui tirent leur existence du financement apporté par des annonceurs. Les premières, de manière classique, dépendent de leur capacité à générer de la satisfaction par l'offre d'une utilité résultant de la consommation d'un bien symbolique. En revanche, un paradoxe réside au plus profond du fonctionnement des chaînes financées par la publicité : ce qui pousse les producteurs à

1 Ch. DuChet, "Le statut de la publicité et la multiplication des chaînes", Communications, $\mathrm{n}^{\circ} 51,1990,153-174$, p. 168. 
investir des sommes considérables dans la promotion, c'est en effet ce que nous appellerions une rareté de la rareté. Si le désir est infini, son expression dans les formes d'objets de consommation atteint rapidement un seuil de saturation qu'il s'agit pour les producteurs de relever

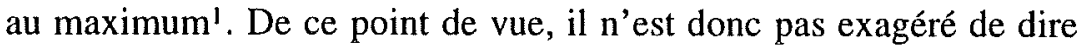
que ce qui permet aux chaînes financées par les annonceurs de survivre, c'est leur capacité à créer de l'insatisfaction sur le plan réel, de la vie quotidienne. Or, cette capacité n'existe que dans l'exacte mesure où ces chaînes parviennent à attirer le spectateur en lui offrant une satisfaction sur le plan symbolique -ce que symbolise le GRP. Nous tenterons plus loin de cerner les enjeux de ce mode différent de fonctionnement.

Pour l'instant, contentons-nous de constater que la nécessité de maximiser les taux d'audience, devenant le seul critère rationnel d'évaluation des émissions de "produits télévisés", conduit les diffuseurs à se soucier anxieusement des goûts du public en matière symbolique. Ces goûts sont pris dans leur actualité, c'est-à-dire qu'ils sont résumés dans une demande fondée sur des préférences ne faisant l'objet d'aucune interrogation; nous retrouvons ici un des postulats centraux de la compréhension micro-économique des agents consommateurs: celui de leur transparence à eux-mêmes, du recoupement parfait de la demande exprimée et du manque sous-jacent. S'agissant d'offrir aux consommateurs ce qu'ils demandent, deux pistes s'ouvrent, qui correspondent selon nous à la double définition de la notion d'intérêt dans le sens commun. La première s'inscrit sur l'axe de l'actualisation, le long duquel les expériences concrètes, les situations vécues sont traduites et représentées comme les matérialisations d'une définition du sens; l'intérêt pour un produit, dans ce sens, c'est la prise de conscience que ce produit est la forme concrète et tangible de ce dont j'ai besoin, ce qui pour moi réalise l'expérience phénoménologique de la satisfaction. La seconde définition de l'intérêt s'inscrit sur l'axe de la médiation, qui voit des situations vécues plus ou moins négativement du point de vue de l'actualisation prendre sens en ce qu'elles sont véhiculées par des médiums hors de leur sphère de production afin de produire des performances sur des sphères d'aboutissement où l'actualisation peut avoir lieu; l'intérêt

1 Ce lien entre financement des chaînes et société de consommation est établi par N. GanRham, "Les limites de la théorie du marché", in F. Du Castel, P. ChAMBAT \& P. MUSSO (eds.), op. cit., 281-295, p. 284. 
pour un produit, dès lors, résulte de la capacité de ce produit à me servir à atteindre la satisfaction en-dehors de lui-même, en tant que moyen'. Sans entrer dans les détails, nous pouvons classer dans la première catégorie tout ce qui touche au divertissement, et dans la seconde ce qui se rapporte à l'information. On pourrait poser l'hypothèse que les actualisations sont généralement préférables, du point de vue du sens, aux médiations qui comportent des "pertes" - de ce point de vue, la médiation temporelle (c'est-à-dire l'inscription dans une séquence sacrifice-récompense, telle qu'elle se manifeste dans la formation par exemple) est plus risquée encore que la médiation spatiale (c'est-à-dire l'inscription de cette séquence entre des lieux investis concomitamment, comme le travail et la famille par exemple). Cette hiérarchisation peut permettre de comprendre les priorités accordées au divertissement sur l'information et, en dernier lieu, la formation.

La spécificité du produit audiovisuel par rapport aux autres produits est qu'il se situe d'emblée dans l'ordre symbolique. Si rareté de la rareté il y a -et c'est le cas dès lors que des chaînes concurrentes tentent d'attirer à elles le public le plus large possible-, il incombe au produit lui-même de susciter la demande à laquelle il répond. C'est à cela que nous nous référons quand nous soulignons le caractère autopromotionnel du discours médiatique. Or, comme mentionné plus haut pour les produits matériels, la rareté doit se comprendre moins comme simplement manque que comme expressibilité de ce manque dans les catégories proposées par une offre. L'enjeu devient dès lors pour le discours médiatique de s'imposer comme système de catégories susceptible de résorber en entier l'insatisfaction. Cette définition correspond assez bien à celle de la néo-télévision, dont la caractéristique la plus frappante est d'être autoréférentielle, sans cesse renvoyant à elle-même comme si la consommation télévisuelle devait suffire à remplir l'existence. "[P]asser de la paléo- à la néo-télévision, c'est passer d'un fonctionnement en termes de contrat de communication à un fonctionnement en termes de contact; du coup, se trouve également abolie la séparation entre espace de la réalisation et espace de la réception : à la néo-télévision, tout se passe à l'intérieur d'un

1 Ces deux concepts sont développés théoriquement dans un autre contexte (celui du travail peu qualifié) par E. Belin, Th.-M. Bouchat, Ph. Cornil et B. Delvaux, Le sens au quotidien. Recherche avec des jeunes femmes en situation de précarité, Bruxelles, Services fédéraux des affaires scientifiques, techniques et culturelles, 1995. 
même espace télévisuel qui se confond lui-même avec l'espace quotidien"l. Le "tautisme" de L. Sfez va dans ce sens également. Mais le fait le plus frappant de cette auto-référentialité, c'est le ton laudatif sur lequel elle se conjugue; on pourrait dire que toute la production audiovisuelle n'est qu'une longue publicité pour l'audiovisuel.

\subsection{Transition par les théories des effets profonds}

S'agissant de cerner les conséquences de cette logique sur l'espace public, J.-M. Ferry fait partir son raisonnement de la notion d'agenda-setting: "la nouvelle idéologie insinue une dépolitisation profonde des enjeux de la communication publique, telle qu'elle est organisée, dans nos sociétés complexes, sous la dominance du principe médiatique. L' «autonomie du public» consacrée dans la sociologie des médias, se laisse sans peine relayer, dans l'idéologie du système, par l'«autonomie de la programmation» qui ne connaît que la loi du public, celle de son plaisir dont les programmateurs/marketeurs/médiateurs sont les oracles professionnels. En proclamant cette autonomie, l'idéologie nouvelle tente d'immuniser le pouvoir de programmation contre la discussion publique. (...) Comment rendre réflexifs les critères de sélection qui interviennent d'abord au niveau de la programmation? Tel peut être le point de départ problématique d'une théorie critique des médias aujourd'hui"2. Et certes, une telle entrée en matière pourrait révéler des biais éditoriaux introduits par la régulation marchande. Deux tendances pourraient ainsi être relevées : la première marquerait la primauté de l'information locale sur l'information nationale et internationale, témoignant d'un souhait de raccourcir les distances entre réalité et représentation sur l'axe de la médiation; la seconde indiquerait la suprématie des thématiques ayant une dimension ludique sur les sujets "sérieux", traduisant un investissement sur l'axe de l'actualisation directe. L'objectif est clair : Ferry "pense au poids des limitations structurelles internes d'une communication médiatique visant à mode-

1 Fr. CAsetTi \& R. Odin, "De la paléo- à la néo-télévision. Approche sémiopragmatique", Communications, $\mathrm{n}^{\circ}$ 51, 1990, 9-26, p. 20.

2 J.-M. FERRY, Les enjeux de la liberté communautaire dans l'espace public moderne, ronéoté, 1993, p. 20. Voir aussi: J.-M. FERRY, Philosophie de la communication. 2. Justice politique et démocratie procédurale, Paris, Ed. du Cerf, coll. Humanités, 1994, p. 32-34. 
ler le sens commun et l'opinion publique, non pas tant par l'instillation de modèles de comportement que par la sélection peu critique des thèmes portés à l'attention du public. Pouvoir immense, pouvoir exorbitant, s'il n'est pas domestiqué, que celui de décider quasi souverainement ce qui mérite d'être rendu public, et ce qui, à l'inverse, n'est pas digne de Publicité"l.

Pourtant, si cette exigence de procéduralisation des choix éditoriaux ne peut guère qu'être jugée légitime, on peut se demander si la démarche, du simple fait qu'elle soit entreprise, n'évacue pas trop précipitamment la possibilité d'une relation, peut-être médiate, entre l'intérêt du public et l'intérêt public. Nous retrouvons ici les problèmes évoqués plus haut, relatifs à la définition de la publicité. Le point de vue complémentaire à cette réflexion qui assure une préséance du normatif sur l'analytique nous semble pouvoir être illustré par l'approche d'A. Ehrenberg, traitant des reality shows. Pour lui, le succès de ces émissions réside dans ce qu'elles expriment des problèmes qui, considérés comme privés dans des sociétés confrontés au problème collectif majeur de la rareté matérielle, peuvent progressivement prétendre au statut de problèmes publics si on admet que la rareté matérielle se complète aujourd'hui d'une rareté identitaire"2. "La «télévision des réalisateurs» considérait son public populaire comme une classe d'élèves, la télévision de délassement le voyait comme une foule de consommateurs dans un supermarché, la télévision de communication le traite comme un individu en difficulté : comment s'approprier un monde incertain? Un code culturel dominant auquel on n'a pas accès? (...) Comment construire des identités individuelles quand les identités collectives sont fragilisées?"3. Certes, il y a quelque inconvénient à présenter ainsi la télévision de "communication" comme une étape ultérieure à celle de "consommation", alors qu'elle n'en est manifestement qu'une forme spécifique, et la confiance dans les potentialités réelles de résolution de problèmes personnels par de telles mises en scènes tient, comme le relève Gérard Leblanc ${ }^{4}$, du mythe ; faut-il pour autant considérer le "massage médiatique" comme une simple idéologie?

1 J.-M. FerRY, préface de B. LiBoIs, L'éthique de l'information, op. cit., p. XII.

2 Le terme est de J. DE MUNCK, "L'aide psycho-affective: l'état social dépassé?", Revue Nouvelle, 85 (1992), p. 30-43.

3 A. EHRENBERG, "La vie en direct ou les shows de l'authenticité", Esprit, n 1, 1993, p. 30.

4 G. Leblanc, "Happy ending? Scénarios de la vie ordinaire", ibidem, p. 48. 
Une autre critique formulable à ce programme de recherche en termes d'effets puissants concerne son caractère limité; des catégories comme l'infotainment indiquent déjà l'importance de la manière dont on traite les thèmes, au-delà de leur sélection. L'ouverture de la dimension publique de la télévision hors des limites de l'information apparaît, avons-nous dit, comme essentielle. Enfin, la prise en compte du caractère fluent et des effets profonds semble aller de pair avec une théorie critique pertinente des médias. À cette proposition de prise en charge par une instance paritaire indépendante des critères de sélection des sujets et des modes de réalisation des "scénarii" des journaux télévisés, il convient dès lors, nous semble-t-il, de tenir la porte ouverte à d'autres manières de poser le problème du nouvel espace public.

\subsection{Transition par les théories des effets profonds}

S'il existe un mécanisme logique par lequel la régulation marchande de la production audiovisuelle tend à favoriser l'émergence d'une rhétorique auto-promotionnelle, et si l'examen empirique des formes de présentation de la réalité semble confirmer la prééminence de ce type de mise en scène, alors il convient de se pencher sur la relation existant entre cette néo-télévision et les formes de rapport au social et à l'histoire des consommateurs pris comme citoyens.

On a beaucoup écrit sur l'influence de l'introduction de la publicité sur le climat programmatique d'ensemble d'une chaîne. Le point essentiel ici est l'établissement d'un lien entre le contexte télévisuel et l'espace publicitaire. Dans la logique du produit dual, en effet, ce lien conditionne à lui seul toute la logique du contrat passé entre l'annonceur et le diffuseur: si ce dernier prétend vendre l'accès au public, c'est parce que le public attiré par les produits télévisuels est rendu disponible aux messages promotionnels qui, eux, ne sont pas des produits télévisuels. Cette équivalence a été longtemps évidente: entre les deux types d'émissions, la relation était établie par la proximité temporelle, tout simplement. La juxtaposition des régimes était permise par une relative rémanence de l'audience. Pourtant, cette forme relativement précaire et maladroite s'est progressivement révélée être le talon d'Achille de la machinerie financière ; la diffusion des téléviseurs avec télécommande, associée à la pratique du zapping, 
révèle cette faiblesse extrême 1 . Le public s'avérant labile, surtout lorsque se multiplient les interruptions publicitaires, il importe d'assurer par des formes plus fiables sa réelle disponibilité ; c'est ainsi que, comme le montre $\mathrm{A}$. Vernet, on assistera à une fictionnalisation des spots publicitaires, afin que la rupture avec leur contexte de diffusion soit moins criante. L'affirmation doit être complétée eu égard à l'intérêt des diffuseurs eux-mêmes, qui est d'attirer les annonceurs; on peut dire par conséquent que ce processus de transformation des messages publicitaires doit être compris plutôt comme un brouillage de la frontière entre registres. Cette fusion peut prendre de multiples formes : qu'il s'agisse du lardage des fictions, de l'accélération exponentielle des rythmes des séquences, du bartering, du sponsoring, des "clip-pubs", des fiction-pubs et des téléromans ou encore des advertnewcasts, il s'agit toujours de réduire l'hiatus séparant l'encart de l'encadrement. S. Daney repère dans le champ du cinéma cette hégémonie de la forme publicitaire: pour lui, les formes rhétoriques issues de ce registres servent dorénavant de trame narrative à toute communication médiatique ${ }^{2}$. La notion de "discours publicitaire" de Laurent Gallissot va plus loin encore: ce qui se jouerait, c'est une assimilation réciproque des cadres de l'expérience et des formes publicitaires ${ }^{3}$. Restent à comprendre les raisons de cette colonisation. Pour Graham Murdock, dans une optique intentionnaliste, les annonceurs "ont assez peu de contrôle sur l'environnement programmatique qui entoure leurs messages, et avec la montée des télécommandes, les publics peuvent éviter les plages publicitaires en passant d'une chaîne à l'autre. La solution est d'intégrer le message promotionnel dans les programmes eux-mêmes. (...) Considérées ensemble, ces initiatives assurent que les espaces publics offerts par la télévision sont substantiellement colonisés par la parole commerciale et le discours du

1 C'est ce qui explique l'effet de "panique" déclenché par l'annonce, au début des années ' 80 , du phénomène: le postulat de régularité de l'audience s'avérait n'avoir été vérifié que par effet d'imprécision des instruments de mesure d'audience (que le phénomène ait eu ou non un fondement réel importe peu : ce qui est prouvé ici, c'est l'importance du postulat). Cf. D. ChATEAU, "L'effet zapping", et M. VERnET, "Incertain zapping", deux articles du numéro déjà cité de Communications.

2 S. DANEY, Devant la recrudescence des vols de sacs à main, Paris, Aléas, 1991, p. 163.

3 L. Gallissot, "La signification culturelle de la publicité", Communication, 14 (2), 1993, p. 14. 
consumérisme" . Cette distinction entre "parole commerciale" et "discours consumériste" est plus importante qu'il n'y paraît ; elle laisse entendre qu'au-delà des messages promotionnels précis, c'est une publicité pour la consommation qui imprègne le flot télévisuel. Ce climat est logiquement déductible du mode de financement des chaînes à produit dual. Nous pouvons montrer que, plus profondément encore, il résulte de manière presque automatique de la logique marchande, que celle-ci se réalise par le dispositif institutionnel de la publicité ou par l'appareillage technique de la télévision payante. Nous avons en effet indiqué que le produit télévisuel est alors conçu comme produit de consommation, porteur d'une utilité résultant de son aptitude à répondre à une insatisfaction, mais qu'en même temps il est à comprendre comme un discours destiné à présenter ce mode de résolution de l'insatisfaction comme voie royale vers la satisfaction. En quoi, toutefois, consiste exactement la consommation médiatique, sinon en une consommation de symboles? Cette consommation de symboles est sans doute partiellement réductible à un bien-être matériel -se reposer dans le fauteuil, se laisser bercer par la logorrhée; mais l'essentiel de l'utilité procurée nous semble résider précisément dans le contenu même du produit médiatique. Nous définirons dès lors la consommation symbolique comme la résolution des insatisfactions sur le plan symbolique. Autrement dit, présenter l'image comme bien de consommation implique de la configurer comme mise en scène de la satisfaction -car la satisfaction symbolique ne peut résulter d'autre chose que de l'identification à une satisfaction (au sens très large) présentée comme réelle, c'est-à-dire mimétique. Telle est la seule valeur d'usage possible des biens symboliques. Le discours auto-promotionnel consiste à vanter ce mode particulier de consommation symbolique.

La mise en scène de la satisfaction n'implique pas en soi un discours consumériste : s'il est vrai que le schéma consumériste n'est qu'une mise en forme socialement et historiquement constituée du rapport au désir, rien n'empêche que soient mis au point des scénarios insistant sur les apories de ce construit et sur la possibilité de solutions alternatives. Trois faits viennent cependant refermer cet éventail de possibilités. Le premier résulte de l'enchâssement mentionné du

1 Gr. MURDOCK, "Citizens, consumers, and public culture", in M. SKOVMAN \& K.C. SCHRøDER (eds.), Media cultures. Reappraising Transnational Media, London New York, Routledge, 1992, p. 36-37. (traduit par nous). 
discours et du méta-discours nécessité par la promotion de la consommation médiatique: ce télescopage a pour effet que en tant que métadiscours, le discours médiatique est de nature consumériste, posant l'acquisition du produit médiatique comme voie vers la satisfaction; insister trop lourdement sur la possibilité d'ordonnancements alternatifs du désir serait, dans un tel contexte, prendre le risque d'un discours auto-dénigrant ${ }^{1}$. Le second tient à ce que les schémas consuméristes sont culturellement prédominants et semblent s'être constitués en rhétorique quasi naturelle de la présentation médiatique de la réalité; ils fournissent un stock de clichés mobilisables plus facilement que n'importe quel autre discours. Enfin, lorsque les chaînes financées par la publicité tentent d'attirer à elles les annonceurs, elles ont intérêt à s'opposer aussi peu que possible aux schémas qu'ils utilisent dans leurs messages publicitaires. Or, ces schémas sont construits entièrement sur une magnification de la solution consumériste, c'està-dire sur une présentation de celle-ci comme point de jonction entre principe de réalité et principe de plaisir ${ }^{2}$. Ce que les publicitaires laissent transparaître dans leurs spots, qui sont comme des "saturés sémantiques"3, les diffuseurs n'ont qu'à le reprendre sous une forme euphémisée. Ces éléments montrent que la télévision publicitaire est plus que la télévision payante sujette à l'hégémonie du discours consumériste : cette dernière peut en effet, par une stratégie de marketing, s'agencer sur une remise en question des formes dominantes pour en faire un argument de vente; elle réalise ainsi le verdict prophétique d'Adorno: "[i]l a été prévu quelque chose pour chacun afin que nul ne puisse échapper, les différences sont mises en relief et diffusées partout. Le fait d'offrir au public une hiérarchie de qualités n'a pour but qu'une quantification d'autant plus parfaite"4.

1 Notons qu'en toute logique, une contradiction demeure entre le discours promotionnel des annonceurs et celui des médias: le premier donne en effet une version avant tout matérialiste du schéma consumériste, alors que le second insiste sur sa dimension symbolique. Cette antinomie peut être représentée par le cas de figure où la consommation télévisuelle s'avérerait telle qu'elle remplacerait la consommation de produits matériels.

2 J.-Cl. SoulaGES, "Les récits du monde", Mscope, nº 8, 1994, p. 85.

3 H. BOYER, "Le spot comme marché", ibidem, p. 69.

4 Th. W. ADORNO \& M. HORKHEIMER, "La production industrielle de biens culturels. Raison et mystification des masses", in La dialectique de la Raison, Paris, Gallimard, 1974 (1947), trad. E. Kaufholz, p. 132. 


\section{Régulation de l'audiovisuel : viser juste}

L'avantage d'une approche des effets en termes de fonction d'agenda et de méthodologie journalistique couplée à une estimation des risques focalisée sur la sauvegarde des thèmes d'intérêt public ainsi que la garantie d'un pluralisme axiologique, réside dans leur caractère opérationalisable. L'inconvénient est qu'on risque à s'y prendre ainsi de laisser dans l'ombre les aspects les plus profonds qui dessinent le rapport du citoyen à l'espace public. Cette complexité, cette impossibilité de définir $a$ priori et in abstracto les solutions à apporter, les portes à entrebâiller et les cadenas à poser, les peines et les sanctions à distribuer, est un phénomène fondamental du droit contemporain, comme l'indique Jacques Lenoble'. Dans un tel contexte -et nul ne niera que le monde des médias en offre un exemple d'école- le législateur ne peut plus prétendre construire un modèle de la réalité pour ensuite promulguer des normes abstraites: non seulement les effets pervers de telles réglementations les rendraient inefficientes, mais de plus, il n'est pas possible de savoir réellement ce qu'il faudrait réguler -la tentation devenant grande, avons-nous dit, de ne s'occuper que de ce qui est régulable: droit de propriété, contenus diffusés, quotas d'informations. Mais si le sens du texte médiatique est à comprendre sémio-pragmatiquement, si le ton joue plus que le contenu, si les trames narratives interviennent bien plus profondément que les images, comment s'y prendre? L'idée d'une autorégulation par le milieu professionnel est écartée avec force d'argumentations par B. Libois², et l'hypothèse d'une intervention régulatrice par des instances de formation permanente recèle l'inconvénient de ne pouvoir qu'opposer les logiques systémiques de fonctionnement à la volonté individuelle des journalistes. De fait, comme le montre J. De Munck, la question de la régulation des médias renvoie à celles, plus profondes, qui concernent la possibilité de formes démocratiques du lien social dans une société déformalisée: "[1]a première est celle des conditions d'articulation des questions publiques, c'est-à-dire l'organisation d'un espace public adapté à la nouvelle situation. La seconde est celle d'une transformation du droit, qui se distingue du formalisme de la première modernité et de la matérialisation propre à l'État social. La troisième question porte sur

1 J. Lenoble, Droit et communication, Paris, Éd. du Cerf, 1994,.p. 10.

2 B. LiBoIs, L'éthique de la communication, op. cit. 
la réorganisation de l'État pour faire face à ses nouvelles missions"'. La scission sémantique entre service et secteur public, ainsi que la possibilité d'une délégation des compétences réglementaires à des autorités administratives indépendantes, toutes deux encouragées par Libois, s'acheminent assurément dans ce sens.

Cette reconsidération de la part du flou dans le langage juridique nous semble conférer une place nouvelle à des "sfumatos" théoriques tels que ceux qui précèdent en même temps qu'elle en tire des éléments de légitimité. Trop souvent, dans le langage juridique, les finalités de la régulation s'effacent derrière la méthodologie; or, cette émancipation du droit positif par rapport aux arguments du monde vécu dans lequel il s'enracine est inefficace en même temps qu'illégitime $^{2}$. C'est qu'en effet, à force de fourbir le droit positif, d'en aléser les dispositions du point de vue exclusif de la cohérence interne, les institutions sociales courent le risque de n'assurer leur emprise sur l'historicité que par des biais protocolaires et désincarnés, délestant du même coup les ajustements sociaux empiriques de toute exigence de rationalité. C'est ce recyclage des potentialités du monde vécu que rend possible la solution procédurale: des instances régulatrices dont le principe premier de fonctionnement serait la concertation entre les acteurs concernés sont à même de rendre au droit sa légitimité inductive, à condition toutefois que des mesures soient prises pour éviter la confrontation de particularismes et de corporatismes - ce qui implique une théorie de la mesure comme système d'attribution de valeur argumentative en fonction de critères consensuels. L'intérêt de J.-M. Ferry pour les théories de l'agenda setting s'ancre dans un tel souci: si les modèles de seringue hypodermique sont falsifiés empiriquement, cela signifie que la régulation des contenus n'a aucune raison d'être. En indiquant que ces effets puissants cachent peut-être d'autres logiques influençant le rapport au politique, nous prenons le parti de profiter de l'assouplissement des structures procédurales de réglementation pour désigner des points du débat à ne pas laisser dans l'ombre, et engageons une discussion sur les modes adéquats de régulation de ces dimensions sans laquelle les instances de régulation se transformeront inévitablement en cellules de morale aussi inefficaces que bien-pensantes. Ainsi, l'inscription de missions

1 J. DE MuNCK, "Déformalisation, dérégulation et justice procédurale", Les Carnets du Centre de Philosophie du Droit, ${ }^{\circ} 10, \mathrm{p} .11$.

2 Cf. à ce sujet J.-M. FERRY, "Le complexe socio-politique", in Les puissances de l'expérience, tome II, Paris, Éd. du Cerf, 1991, p. 41 à 50. 
minimales de service public dans le cahier des charges de chaînes privées doit-il tenir compte des logiques culturelles de fonctionnement des organisations médiatiques et de l'impossibilité de définir en termes formels systématiques ce en quoi consisterait le respect de telles exigences.

Deux lignes de raisonnement nous semblent valoir d'être suivies. La première, d'application dans les chaînes de service public, consisterait en une tentative d'opérationalisation des diverses formes de rationalité régissant l'activité de production des médias au niveau quotidien, qui ne repose pas sur des postulats idéalistes. Trop souvent, la politique d'incitation se limite à son aspect financier, quand les mesures pénales sont mises en œuvre en dernier ressort par la violence physique ; un examen des motivations des acteurs en situation concrète révèle pourtant que le fonctionnement des champs réels repose sur la mobilisation de capitaux très variés et partiellement convertibles seulement, tels le prestige, le pouvoir, l'influence, ou encore l'actualisation de principes de sens. Il est de coutume de préciser que l'impératif économique est, dans le service public, une contrainte et non un objectif; une réelle confiance dans les logiques culturelles pourrait conduire à la mise en place de formes institutionnelles efficaces qui établiraient une spécificité des chaînes publiques par rapport aux autres -et par là à l'instauration d'un "pluralisme des rationalités"1. La seconde ligne de raisonnement, peut-être plus adéquate lorsqu'on considère les entreprises privées ${ }^{2}$, concerne l'introjection au sein même de la rationalité économique des externalités. Comme le montre A. Lipietz ${ }^{3}$, le développement industriel de la société de production, puis de consommation, n'a jamais résulté de l'application stricte de la rationalité économique short-termist des agents économiques, mais de l'émergence en mineure de cette rationalité d'une "conscience" susceptible d'en

1 Plutôt que d'une critique idéaliste de l'argent, à laquelle on peut opposer des arguments de type simmelien, nous cherchons à prendre acte du caractère limité de cette forme construite pour répondre aux exigences d'une sphère particulière de l'activité humaine et insuffisante pour la régulation d'autres.

2 Quoique, comme le prouvent les multiples formes de la corporate culture, le dynamisme de ces entreprises les ait conduites à abandonner le postulat d'une linéarité de l'efficacité des stimulations économiques pour élargir le spectre des incitants, dans un contexte pourtant marqué par un pragmatisme économique des moins sentimentaux...

3 A. LIPIETZ, Choisir l'audace: une alternative pour le vingt et unième siècle, Paris, La Découverte, 1989. 
élargir les enjeux à l'échelle macrosociale (fordisme) et à long terme. Outre le caractère problématique de l'émergence d'une conscience de telles implications au niveau de la pratique professionnelle, l'obstacle majeur d'une telle extension de la rationalité instrumentale et stratégique réside dans ce que la théorie des jeux appelle le free riding. Il incombe dans une telle situation au pouvoir politique d'établir par des mesures normatives des structures d'internalisation des externalités négatives et positives de leur activité -ce qui à long terme peut conduire à une redynamisation du marché profitable sur la scène internationale. 